1 Universidad Nacional de Entre Ríos (Uner) - Entre Ríos, Argentina. Orcid: https://orcid. org/0000-0002-63528668

agustinabarukel@gmail.com

2 Universidad Nacional de Buenos Aires (UBA) -

Buenos Aires, Argentina. Orcid: https://orcid.

org/0000-0001-9372-

7556

astolkiner@gmail.com

\section{El problema del diagnóstico en salud mental: clasificaciones y noción de enfermedad}

\author{
The diagnosis in mental health: labeling and the concept of disease
}

Agustina Barukel1, Alicia Stolkiner ${ }^{\mathbf{2}}$

DOI: 10.1590/0103-1104201811808

RESUMEN El artículo presenta avances de una investigación de Doctorado en Ciencias Sociales acerca de las prácticas y políticas vigentes en Argentina para el abordaje de los padecimientos mentales. Se trata de un estudio de caso con perspectiva etnográfica, realizado en un hospital público monovalente de la provincia de Santa Fe. Aborda las cuestiones referidas al problema de la clasificación de los 'pacientes' que cursan internaciones en este tipo de instituciones, a partir del debate al interior de la Psiquiatría y del campo 'psi' al respecto de las nosografías en salud mental para la realización de los diagnósticos. Se evalúa el problema de la multiplicidad de diagnósticos para un único caso y se problematiza la noción 'sin conciencia de enfermedad'. Las técnicas utilizadas para la construcción de los datos son la revisión de historias clínicas, las entrevistas en profundidad, y la observación participante. Las principales conclusiones son que hay una alta prevalencia de casos de superposición de categorías diagnósticas en las personas internadas, y una utilización extendida del concepto 'enfermedad' para referirse a las manifestaciones del sufrimiento psíquico, que desconoce el debate disciplinar en torno a dicha categoría.

PALABRAS-CLAVE Clasificación. Diagnóstico. Enfermedad. Salud mental.

\begin{abstract}
This article presents results of a Social Science PhD research on practices and policies that shape the way mental illnesses are addressed in psychiatric institutions in Argentina. The ethnographic case study placed in a mental health public hospital in Santa Fe specifically analyses the labeling of patients in such an institution, drawing on debates around nosography in mental health. We critically discuss problems around multiple diagnoses and challenge the category 'lack of disease awareness'. The methodological techniques we employed in this research include the review of medical record, in-depth interviews and participant observation. We found high prevalence of diagnostic category overlap and an excessive use of the concept of 'disease' to refer to psychic suffering, which stands in stark contrast to the debates around this category that have been developing in the field.
\end{abstract}

KEYWORDS Classification. Diagnosis. Disease. Mental health. 


\section{Introducción}

Es difícil conocer con exactitud cuántas instituciones de internación de salud mental existen en Argentina, y cuál es la población que atienden. Los números son desconocidos por varios motivos: un escaso desarrollo de epidemiología en salud mental; la variedad de instituciones privadas que con diferentes nombres - hogares, geriátricos, casas de medio camino, clínicas, comunidades terapéuticas - cumplen la función de asilar y no son parte de las estadísticas del sector; la discrecionalidad con que actúa el subsector privado; la falta de control con que funciona el sistema público; entre otros. Para 2010, la estimación de la Dirección Nacional de Salud Mental y Adicciones indica que, de la población total mayor a 15 años, el $21 \%$ habían sufrido en el último año algún trastorno mental o del comportamiento. Se trata de casi 6.300.000 personas'. Según el mismo informe, existen 54 instituciones públicas con internamiento crónico repartidas en 15 provincias: 1 colonia nacional, 11 colonias provinciales y 42 hospitales monovalentes provinciales. Se estima que unas 21.000 personas se hallan internadas en estos: $2 / 3$ en las colonias, y las restantes en los hospitales.

La provincia de Santa Fe cuenta con tres instituciones públicas de internación especializadas en salud mental, que suman un total de 530 camas. La institución donde realizamos nuestro estudio de caso representa el $15 \%$ (78 camas), con un porcentaje de ocupación que se mantiene relativamente estable, y ronda el 95\% (74 camas) ${ }^{2}$.

A grandes rasgos, hay tres vías de ingreso a esta institución. Una es a través del Comité de Admisión que funciona en la Guardia y Tránsito. Cuando las personas son enviadas o concurren a la Guardia del Hospital, son entrevistadas por un Comité interdisciplinario que evalúa la situación y determina si la misma requiere de internación. Este criterio está fijado por el principio del riesgo para sí mismo o para terceros estipulado en la
Ley Nacional de Salud Mental 26657. Si ese riesgo existe, la persona es ingresada y le es asignado un equipo tratante. Esas internaciones pueden ser de dos tipos: voluntarias - cuando la persona accede a ellas prestando su consentimiento - e involuntarias. En este último caso hay resguardos legales específicos también contenidos en la Ley; entre ellos, la persona tiene derecho a recibir asesoría legal por un Cuerpo de Letrados o equivalente. La extensión del tiempo de la internación permite distinguir entre pacientes agudos y pacientes de larga institucionalización (cronificados, o casos de revolving door ${ }^{3}$ ). Es necesario aclarar que, si bien la normativa establece que la internación debe ser lo más breve posible, y estar destinada sólo a estabilizar el cuadro para luego continuar el abordaje de manera ambulatoria, persisten las internaciones prolongadas. Para el caso de la institución estudiada, por ejemplo, el 78\% de los casos abarcados corresponden a casos de larga institucionalización, definición para las internaciones con duración de 1 año en adelante.

La segunda es la vía judicial, e involucra personas que están en conflicto con la ley penal. Se trata de personas que provienen del sistema penitenciario y son ingresados al hospital por una orden. Incluso cuando el equipo tratante evalúe la situación y concluya que no hay criterio para que esa persona permanezca internada, la decisión está en manos del juez a cargo de la causa.

La tercera es el caso de las personas que cometieron un delito penal y fueron declarados inimputables. En este caso, el 'paciente' no está estrictamente en el circuito penitenciario, pero sí depende del juez que ha dictado la inimputabilidad hasta que éste determine - a instancias del equipo tratante - si cesa la situación de riesgo y puede darse el alta.

Los diagnósticos psicopatológicos son variados, y aunque hay abismos entre cada uno de los casos en su singularidad, hay algo que los iguala: su estatus de internados ${ }^{4}$.

La clasificación de cada uno de esos pacientes organiza el trabajo cotidiano de los 
equipos, traza las metas de los abordajes o tratamientos, permite proyectar 'expectativas', qué es lo esperable de cada uno y para cada uno. Sobran las técnicas y los criterios para clasificar, incluso muchas veces colisionan entre sí, se superponen, se contradicen. ¿Es el diagnóstico, la patología o el 'cuadro de base’? ¿Es el tiempo de internación? ¿Es la presencia o ausencia de recursos materiales, simbólicos, culturales, afectivos? ¿Es el vínculo o no vínculo con la ley penal?

Distinguimos tres dimensiones en el problema de las clasificaciones; por supuesto, cada una atraviesa a la otra:

1. La dimensión de las nosografías existentes en psiquiatría, a partir de las cuáles se fundan los diagnósticos. Comprende el debate en torno al estatuto del padecimiento mental como objeto médico, y blanco de los procesos de normalización ${ }^{5}$.

2. La dimensión de las técnicas y criterios de clasificación dentro del espacio institucional, que no siempre se vincula al diagnóstico psicopatológico, y es la que organiza el trabajo cotidiano de los equipos intervinientes. En el caso de la institución que estudiamos, es la que distingue entre los 'pacientes' agudos, los de larga institucionalización, los 'pacientes' en conflicto con la ley penal, y los 'pacientes sociales'.

3. La dimensión de las clasificaciones que emergen como resultado de la interacción y los procesos de reconocimiento/desconocimiento entre los mismos 'pacientes' internados. Se trata de atender a las construcciones que los mismos usuarios del Hospital hacen de sus compañeros y compañeras, que tienen una función y un efecto clasificador.

Por cuestiones de espacio, desarrollaremos aquí sólo la primera dimensión, que operacionalizamos a través de dos indicadores:

1. La recurrencia de varios diagnósticos superpuestos en las historias clínicas de los 'pacientes' internados; y

2. El uso de la categoría 'sin conciencia de enfermedad' en las evaluaciones de los equipos tratantes.

\section{Material y métodos}

Este trabajo se inscribe en la corriente de investigación cualitativa en salud ${ }^{6}$, que se orienta al análisis de los actores y las instituciones de salud al respecto de:

(a) valores culturales y representaciones sobre su historia y temas específicos; (b) relaciones entre individuos, instituciones y movimientos sociales; (c) procesos históricos, sociales y de implementación de políticas públicas y sociales ${ }^{7(16)}$.

Se trata de un estudio de caso ${ }^{\mathbf{1}}$ que adopta la perspectiva etnográfica ${ }^{9}$ para la recolección/construcción de datos, priorizando la utilización de tres técnicas: las entrevistas en profundidad a profesionales y 'pacientes' de la institución, la observación participante y su sistematización en notas de campo, y el trabajo documental de revisión de historias clínicas.

Para preservar la identidad de los 'pacientes', se utilizan nombres de fantasía y para el caso de profesionales, se indica su profesión entre paréntesis. En todos los casos de entrevistas ha sido utilizado el consentimiento informado, diferenciando su forma entre funcionarios públicos y cuadros técnicos del área de salud mental, trabajadores y usuarios. Ya que no se trata de una investigación con aspectos clínicos, el uso de consentimiento es un requisito fundamentalmente ético ${ }^{10}$, anclado en la perspectiva de defensa de los derechos de las personas con padecimiento mental.

Por último, utilizamos la palabra 'pacientes' entre comillas simples en todos los casos porque es la denominación que se utiliza en la institución para referirse a 
las personas allí internadas, pero no acordamos con dicha categoría.

\section{Resultados y discusión}

Sobre la recurrencia de varios diagnósticos superpuestos por 'paciente'. Llevamos adelante la revisión de un total de 54 historias clínicas de personas ingresadas en el área de Internación del Hospital. En 17 casos - 31,4\% - personas han sido diagnosticadas con dos cuadros psicopatológicos. En 7 - 13\% - con más de dos. Se trata entonces de casi la mitad de los casos considerados (44,4\%). En esta institución se utiliza como referencia la clasificación propuesta por la Organización Mundial de la Salud (OMS) en el capítulo 5 de la Clasificación Internacional de Enfermedades (CIE-10), 'Trastornos mentales y del comportamiento’. El diagnóstico de mayor recurrencia es el de esquizofrenia, seguido por las psicosis en diferentes variantes.

La medicalización como proceso estructurante del gobierno de las poblaciones ha sido objeto de profundo interés para las ciencias sociales. La psiquiatrización es la forma que asume la medicalización en el caso de la locura. La Psiquiatría como rama de la medicina es tributaria de este proceso, le debe la vida, es su razón de ser.

Respecto de la(s) teoría(s) en que se sustenta la Psiquiatría hay fuertes debates al interior de la disciplina. Hay dos puntos a partir de los cuáles es posible pensar la multiplicidad de corrientes que existen a su interior, oportunamente señaladas por Stagnaro"1: la primera, en torno a las nociones de enfermedad y síntoma, de la cual derivan modelos psicopatológicos distintos. La corriente que se funda alrededor de la noción de síntoma, inaugurada por la tradición francesa de Esquirol, discípulo de Pinel, es una corriente a-teórica: no desarrolla teoría, sino que describe síntomas, y pone en entredicho la posibilidad de hablar de 'enfermedad mental' en tanto entidad mórbida de origen orgánico. La corriente alemana que encabeza Emil Kraepelin, por otro lado, parte de la noción de enfermedad, y sostiene que es posible hallar entidades de origen natural, similar a otras enfermedades del cuerpo, con etiología propia. Aunque postula eso como ideal, no es capaz de señalar las causas y el origen de las enfermedades de la mente y del comportamiento, por lo que se dedica más bien al estudio de su evolución y de sus principales características.

La segunda es que en toda práctica psi hay subyacente una noción del funcionamiento mental, del funcionamiento del ser humano y de sus cualidades ${ }^{11}$. Por lo que las diversas ideas sobre el inconsciente, la conducta, la relación cuerpo-mente, entre otras, también derivan hacia nociones diversas del padecimiento, que se diferencian epistemológica, teórica y prácticamente. De ahí las diferencias en diagnosticar y en definir tratamientos o abordajes.

Estos dos puntos - dos modelos psicopatológicos distintos, uno en base a la noción de síntoma y otro a la de enfermedad; una teoría subyacente sobre el funcionamiento mental en cada caso-, dan por resultado la imposibilidad de acuerdos y consensos hacia el interior de la psiquiatría respecto de su objeto. Paradójicamente, la cantidad infinita de enfermedades o síndromes mentales clasificados desde el siglo XVIII a la actualidad no son muestra de su fortaleza sino más bien de su debilidad ${ }^{\mathbf{2}}$.

En las revisiones de las historias clínicas que se han llevado adelante, llama la atención la recurrencia de la superposición de diagnósticos -en algunos casos, entre 4 y 6 - por 'paciente'. ¿Cuántas clasificaciones posibles existen para un conjunto de síntomas o de manifestaciones del sufrimiento que presenta una persona? ¿Cómo diferenciar, pero, sobre todo, para qué, una psicosis esquizofrénica de una esquizofrenia psicótica sin que se trate simplemente de una alteración en el orden de los factores?

El desarrollo de la nosología (nosos: 
enfermedad; logos: ciencia, tratado) y la nosografía (nosos: enfermedad; graphein: descripción) en Psiquiatría responden a una necesidad y a una limitación: se necesita del estatuto de ciencia, a la vez que hay una imposibilidad de construir teoría. Como afirma Galende $^{13}$, la psiquiatría hace de la nosografía su única teoría. A la manera en que la anatomía patológica disecciona el cuerpo en busca de sus parámetros normales y sus malformaciones mórbidas, la Psiquiatría intenta en espejo diseccionar las partes que 'faltan' a la personalidad. El resultado es infinito, pero siempre carece de valor científico, al menos al modo en que la ciencia positiva establece los parámetros para ser tal: no hay correlato anátomo-clínico sobre el que sustentar los síndromes construidos. La entidad mórbida en psiquiatría es, a todas luces, un constructo artificial.

Respecto de los cambios y la superposición de diagnóstico en los casos mencionados, varias explicaciones son posibles: la posibilidad de modificaciones del síndrome de acuerdo a su evolución, la reacción al tratamiento, $\mathrm{u}$ otros datos propios del sujeto que padece - su edad, los 'avatares de la vida' -, o una combinación de todo eso; la perspectiva de los diferentes profesionales que tratan a una persona puede ser distinta; un psiquiatra puede corregir el diagnóstico de otro que haya cometido un error; la forma de clasificar un padecimiento puede cambiar en función de nuevos descubrimientos a lo largo de la vida de un paciente; pueden asignarse diagnósticos lo suficientemente generales como para hacer posible que el paciente sea beneficiario de alguna asignación social (pensión, certificado de discapacidad $\mathrm{u}$ otro); también es posible que el paso del tiempo otorgue mayor claridad a quien diagnostica para poder 'ver' manifestaciones de un cuadro psicopatológico.

$\mathrm{El}$ asunto no es poner en tela de juicio la veracidad o no de esas causas; efectivamente puede ser así. Lo que es útil señalar es que, si habláramos de la fractura de un hueso o una hepatitis, no habría debate. Y si hubiese error, habría mala praxis. Si el paso del tiempo permitiese un mejor diagnóstico, la demora podría significar un agravamiento del cuadro, la imposibilidad de una terapia, o de la cura. En salud mental, las implicancias del diagnóstico sobre la vida de la persona son difícilmente medibles en ese mismo plano, lo cuál aún implica que las responsabilidades sobre los avatares diagnósticos no pueden ser las mismas.

En los casos relevados, cada aparición de una nueva clasificación en general no hace referencia a la anterior, ni queda asentado el porqué del cambio en las historias clínicas. Pudimos registrarlos porque aparecen en alguna documentación particular que forma parte de la historia clínica - la evaluación del Comité de Admisión, alguna intervención de las Juntas Especiales que tienen por función intervenir en casos que involucran la salud mental de personas en conflicto con la ley penal, un informe del Servicio de Emergencia o notificaciones a los Juzgados a cargo de las causas de estos 'pacientes', entre otras -, pero no en la evolución diaria que el equipo tratante deja registrado. Tampoco como un cambio deliberado, discutido o fundado en alguna razón o acontecimiento en particular. Ello nos permite ver y problematizar el lugar del diagnóstico psicopatológico en la estructuración de los abordajes.

\section{Sobre el concepto y la experiencia de la enfermedad}

Para estudiar esta dimensión del problema, nos abocamos al estudio de dos casos que componen la muestra. Fernando de 35 años, y Florencia de 29, ambos pacientes del Hospital en que ingresaron en el año 2009, y que en este momento (marzo 2018) están en proceso de externación. Eso quiere decir que ya no están más internados en la institución, pero concurren con frecuencia semanal a 
entrevistarse con sus equipos y retirar la medicación. Como resultado del análisis de sus casos, podemos constatar que la categoría 'sin conciencia de enfermedad' - muy recurrente en el registro de sus historias clínicas - oculta el debate irresuelto en torno al concepto de enfermedad como entidad mórbida objetivable en salud mental.

La noción de 'enfermedad' para referirse al padecimiento subjetivo despierta polémica. En medicina, el concepto de enfermedad se usa para describir una alteración o desviación respecto de un estado normal, que contiene síntomas y signos característicos; se trata de una entidad mórbida que es posible estudiar y conocer en su evolución. En psicopatología, es francamente discutible que aquellas enfermedades que se clasifican, describen, diagnostican sean entidades a las que se pueda otorgar el mismo estatuto que otros cuadros elaborados desde otras ramas de la medicina. En general, cuando se halla un origen biológico de alguna 'enfermedad' clasificada como 'mental' - por ejemplo, la epilepsia -, ésta deja de serlo y pasa a formar parte de otra clasificación - en este caso, de la neurología -. Por eso, las clasificaciones vigentes para el campo de salud mental han optado por el concepto de 'trastorno'. El trastorno es equivalente a 'malestar'; tiene carácter variable, pero, sobre todo, precario en términos científicos. Según Dalal y Sivakumar, citado en Braunstein,

Trastorno [...] es un término a medio camino entre una enfermedad o entidad mórbida y un síndrome en lo que respecta a su consistencia, correlatos y significación ${ }^{12(71)}$.

El Manual Diagnóstico y Estadístico de los Trastornos mentales (DSM-5) de la Asociación de Psiquiatría de EEUU y la Clasificación Internacional de Enfermedades de la OMS son los dos instrumentos a partir de los cuales se clasifican los 'trastornos mentales'. El DSM, tal como su nombre lo indica, comenzó como un proyecto con objetivo estadístico, no clínico. Entre su primera versión, publicada en 1952 y la que se encuentra en vigencia en nuestros días (DSM-5 2013), se han multiplicado las características que diferencian un trastorno de otro de manera espectacular: creció de 106 a 297 categorías psicopatológicas. Las categorías son ayudas para el juicio clínico, y se basan en la suposición - imposible de comprobar, aunque no lo aclare -, que cada diagnóstico específico responde a una también específica raíz patológica.

Este Manual despierta sin dudas muchas polémicas en torno a su utilidad y a sus efectos. Por un lado, hay una mirada que fija un horizonte de esperanza en la molecularización de la psiquiatría propia de nuestros tiempos: una imagen cada vez más certera de la complejidad cerebral, que conlleva una idea cada vez más exacta de las afecciones neurológicas, y a la que es posible corresponder una intervención terapéutica precisa, la de la psicofarmacología ${ }^{14}$. Por el otro, las miradas críticas señalan que esa visión optimista sin embargo no ha hecho aún aportes sólidos para el diagnóstico, pronóstico o tratamiento de las patologías mentales: el conocimiento profundo que tenemos sobre el funcionamiento cerebral sigue sin ser suficiente para darle por fin credenciales científicas a la Psiquiatría. Se sabe asimismo que los momentos previos al lanzamiento de la última versión del Manual estuvieron atravesados por un escándalo al conocerse la injerencia que los laboratorios estaban teniendo para la inclusión de nuevos diagnósticos y la baja de los umbrales en diversas categorías patológicas que requieren, por supuesto, la receta de fármacos.

El Capítulo 5 del CIE-10 taxonomiza los Trastornos mentales y del comportamiento. Incluye 78 categorías, que llegan a 280 con sus desgloses respectivos. Para evitar que quede algo por fuera, recurre a eufemismos tales como 'otros trastornos sin especificar', a la manera de un 'etcétera'. Además del riesgo creciente de patologización de la vida 
cotidiana que promueven, estas clasificaciones viven presas de una paradoja: miles de trastornos clasificados, cuando lo que prima en la definición es la carencia.

Con todo, ya se sabe su debilidad inherente: al no haber manifestación biológica y por ello objetivable de un padecimiento, el diagnóstico en salud mental es a partir de la experiencia siempre individual, única y subjetiva de la persona que sufre. Algo que Canguilhem ${ }^{15}$ señaló hace poco más de 50 años para discutir el concepto positivista de las enfermedades en general, pero que la empresa clasificadora, en particular en salud mental, debe olvidar para seguir existiendo. Por lo tanto, más allá o más acá del concepto enfermedad o la noción de trastorno, hay que recuperar la experiencia del sufrimiento y revisarla epistemológicamente.

Fernando y Florencia son dos pacientes de la institución que llegaron en 2009: Florencia por un intento de suicidio, Fernando provenía del Pabellón Psiquiátrico de un penal provincial, donde estaba por haber cometido un homicidio por el que fue declarado inimputable. Desde su primer ingreso y hasta hoy, Florencia contabiliza más de 16 internaciones; su historia clínica es un relato sostenido sobre el riesgo permanente de dañarse o dañar a otros en el medio en el que vive. Fernando en cambio cumple una condena en los hechos: los años que suma entre cárcel y hospital ya igualan los que hubiese tenido que cumplir si no hubiese sido declarado inimputable. Los registros en la historia clínica muestran el deseo de recuperar por fin su libertad. Un punto en común entre ambos es que, en varios registros de la evolución de sus historias clínicas, aparece la leyenda 'sin conciencia de enfermedad'. La conciencia como atributo de una persona de tener capacidad de sí mismo y de su entorno aparece deteriorada o asechada por una serie de síntomas que, en definitiva, expresan falta de adhesión al tratamiento que propone el equipo e imposibilidad de establecer un curso terapéutico factible para el abordaje de su sufrimiento.

En las entrevistas con estos pacientes, podríamos interpretar la carencia del atributo de conciencia en algunas frases. Por ejemplo, Fernando dice que la combinación de droga e iglesia le causaron un trastorno mental, y que por eso 'cayó en el Hospital'. En realidad, el motivo es que asesinó a un familiar con un cuchillo de cocina, hecho por el que fue declarado inimputable. Él dice que "la jueza me largó porque no podía hacer más nada conmigo"; y que sólo con el tiempo se dio cuenta de que "necesitaba la pastilla".

Quiero ser independiente, quiero hacer mi vida. Pero me di cuenta que la pastilla la tengo que tomar, porque después si no la tomo por 2 o 3 días me siento mal, me vengo abajo.

Florencia en cambio dice que ella sabe que puede ser peligrosa o despertar el temor de los otros: un día, después de varias advertencias, apuñaló a su acompañante terapéutico, también con un cuchillo de cocina, porque la "estaba volviendo loca". No lo mató. Entre risas cuenta que salió en el diario. Pero sus reingresos sucesivos al hospital se explican porque cada vez se retiraba sin el alta, bajo su propia responsabilidad o la de su madre, y ninguna de las dos reconocía la situación de riesgo en que se encontraba. "Yo no estaba loca, no tenía ningún problema, sino que tenía problemas de familia". Pero hay otro dato llamativo: volvía, en general voluntariamente y pidiendo ayuda, a veces a gritos, otras explicando entre risas que "se había tomado unas vacaciones". Pedía insistentemente permanecer en el área de Tránsito, que está separada ediliciamente de los Pabellones de Internación, porque ahí "se siente menos loca y menos desprotegida". Sabe también que necesita la medicación para sostenerse.

Me empezaron a dar otra pastilla para los temblores, y más pastillas, y cada vez más cosas. Bueno, y ahora estoy con el halopidol y sigo nomás con las pastillas; nada más que ahora pareciera que me hacen falta. 
Asumir la toma de medicación aparece, en parte, como 'dar crédito' al menos a una parte de lo que dicen sus equipos tratantes. Lo que sucede con ambos no es que niegan estar enfermos, tienen una propia mirada de lo que les pasa, y por sobre todas las cosas, no quieren estar internados. En el caso de Florencia, la imposibilidad de asumir la necesidad de la internación por riesgo de pasaje al acto requirió de la intervención de un Juzgado de Familia que aprobara la internación involuntaria. En un segundo momento, tuvo que intervenir un equipo interdisciplinario de las Juntas Especiales en salud mental de la provincia, que la derivó a la Colonia provincial. Eso no evitó que siguiera escapándose en varias oportunidades de ambas instituciones. En el caso de Fernando, las fugas fueron durante 7 años su modo de estar en el Hospital.

En este momento, ambos están en proceso de externación, dejan claro que no quieren tener ya más nada que ver con el Hospital.

Fernando: Y bueno, hoy en día estoy tratando de... quiero conseguir un trabajo, hacerme una casa, ya no depender más del hospital.

- ¿a qué vienes al hospital? ¿A atenderte? ¿Te dan la comida también?

Fernando: sí. Pero eso ya no quiero. No estoy de acuerdo con eso, de estar ligado al hospital. No estoy de acuerdo.

- Ahora que estás en proceso de externación, ¿qué tipo de conexión tienes con el hospital?

Florencia: no tengo ningún tipo de conexión. Tampoco quiero tenerla. Es más, no duermo más acá. Ya no duermo más acá, no puedo dormir más acá.

En medicina la noción de enfermedad describe una alteración del estado normal, que reúne un conjunto de síntomas, pasibles de ser estudiados en su estado y evolución. Desde la tradición vitalista, Canguilhem dedicó una serie de estudios al discernimiento de las diferencias entre lo normal y lo patológico, que ponen en entredicho esa aseveración. Allí está la piedra de toque que recuperó Foucault ${ }^{16}$, entre otros, para pensar los procesos de normalización. Dos cosas son importantes de ser señaladas de ese aporte: lo primero es respecto de la normatividad que funda lo normal. Lo que ocurre es que el estado normal que altera la enfermedad no es objetivo, de modo que tampoco puede serlo el patológico. No hay posibilidad de que la enfermedad pueda ser definida en términos fisiológicos y que eso represente algo objetivo. Si se reconoce el carácter normativo - es decir, de establecer normas, parámetros, medidas, valores - de lo normal, la enfermedad ya no es observable como un hecho, sino que se manifiesta respecto de un parámetro previamente fundado, lo cual también la vuelve un valor. Lo segundo entonces es que dicho valor funda una normalidad distinta. La experiencia de lo patológico no es anormal, sino otro tipo de lo normal. Entre salud y enfermedad no existe entonces una diferencia de grado, cuantitativa, sino cualitativa. Estamos hablando de experiencias vitales distintas, no de normales unas y anormales otras.

La consecuencia de poner en entredicho una posible manifestación objetiva de la enfermedad mental es reconocer que ésta se define también en el ámbito de la experiencia individual de quien padece $\mathrm{y}$, que, por eso, lo hace todas las veces de modo particular. De modo que negar la conciencia de enfermedad podría operar como negación de las formas siempre particulares, conflictivas, que colisionan con la de los profesionales, con la de la institución, que cada paciente tiene en la experiencia de su dolencia.

La forma en que un sujeto acepta o niega su enfermedad, la forma en que la interpreta y presta significación a sus aspectos más absurdos, todo esto constituye una de las di-

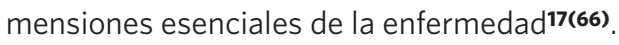




\section{Conclusión o consideraciones finales}

A través del estudio documental de historias clínicas hemos demostrado la alta prevalencia de casos de superposición de categorías diagnósticas en las personas internadas en hospitales monovalentes. A su vez, haciendo hincapié sobre dos casos, hemos puesto en entredicho la noción 'sin conciencia de enfermedad', problematizando la categoría 'enfermedad' para las manifestaciones del sufrimiento psíquico.

Más allá de las limitaciones y complejidades que hemos desarrollado en torno al asunto de las clasificaciones en psiquiatría y salud mental, a la hora de organizar el trabajo institucional, las distribuciones en el espacio y los abordajes, estas clasificaciones y modos de etiquetar las dolencias muestran su sentido práctico. Dicha afirmación es el puntapié a partir del cual abordar otras dimensiones de la clasificación que mencionamos en la introducción - los criterios intra-institucionales que distinguen entre 'pacientes' agudos, cronificados, en conflicto con la ley penal, o con problemáticas sociales y no estrictamente de salud; y las clasificaciones que cada paciente hace de sí mismo y del resto. Son dos puntos que abordaremos en trabajos futuros.

\section{Colaboradores}

Barukel A contribuyó sustancialmente para la concepción y planeamiento y para el análisis y la interpretación de los datos; contribuyó significativamente en la elaboración de la revisión crítica de su contenido; participó de la aprobación de la versión final del manuscrito. Stolkiner A contribuyó significativamente en la elaboración de la revisión crítica de su contenido; participó de la aprobación de la versión final del manuscrito. 


\section{Referencias}

1. Argentina. Ministerio de Salud. Camas disponibles destinadas a la atención de salud mental y egresos hospitalarios del sector público en Argentina. Buenos Aires: DNSMyA; 2010.

2. Gerlero S, Augsburger AC. La salud mental en Argentina: avances, tensiones y desafíos. Rosario: Laborde; 2013.

3. Alberdi J, Mutazzi E, Coll L, et al. La problemática de la institucionalización crónica y el fenómeno de revolving door en pacientes usuarios del Centro Regional de Salud Mental Dr. Agudo Ávila. Rosario: Cátedra Paralela; 2005.

4. Goffman E. Internados. Ensayos sobre la situación social de los enfermos mentales. Buenos Aires: Amorrortu; 1981.

5. Foucault M. La política de salud en el siglo XVIII. En: Foucault M. Saber y verdad. Madrid: La piqueta; 1985.

6. Minayo MCS. Origen de los argumentos científicos que fundamentan la investigación cualitativa. Rev Salud Colect. 2017; 13(4):561-575.

7. Minayo MCS. El desafío del conocimiento: investigación cualitativa en salud. Buenos Aires: Lugar; 1997.

8. Neiman G, Quaranta G. Los estudios de caso en la investigación sociológica. In: Gialdino IV. Estrategias de investigación cualitativa. Barcelona: Gedisa; 2009. p. 213-238.

9. Hammersley M, Atkinson P. Etnografía: Métodos de investigación. Barcelona: Paidós; 1994.
10. Garbus P, Solitario R, Stolkiner A. Aspectos éticos en investigaciones en clínicas en el campo de la salud: Algunas consideraciones acerca del consentimiento informado en personas declaradas incapaces. Anu Investig. 2009; 16:329-338.

11. Stagnaro JC. Nosologías y nosografías psiquiátricas argentinas. Rev Psiquiatría Argent. 2017. 28(133):188-235.

12. Braunstein N. Clasificar en psiquiatría. México: Siglo XXI; 2013.

13. Galende E. Psicoanálisis y Salud Mental: Para una crítica de la razón psiquiátrica. Buenos Aires: Editorial Paidós; 1992.

14. Rose N. Políticas de la vida: Biomedicina, poder y subjetividad en el siglo XXI. La Plata: Unipe; 2012.

15. Canguilhem G. Lo normal y lo patológico. México: Siglo XXI; 2009.

16. Foucault M. El poder psiquiátrico. Buenos Aires: FCE; 2007.

17. Foucault M. Enfermedad mental y personalidad. Buenos Aires: Paidós; 1984.

Recibido en 12/04/2018

Aprobado en 08/08/2018

Conflicto de intereses: inexistente

Apoyo financiero: Facultad de Psicología UBA/UBACyT

20020170100293BA 'Discursos, prácticas, actores y subjetividad

en la articulación de APS y Salud Mental: estudio de caso en investigación social participativa'.

Consejo Nacional de Investigaciones Científicas y Técnicas

Conicet - Argentina 